In dieser Rubrik referieren und kommentieren diese Experten für Sie Arbeiten aus der internationalen Fachliteratur.

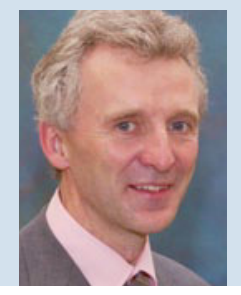

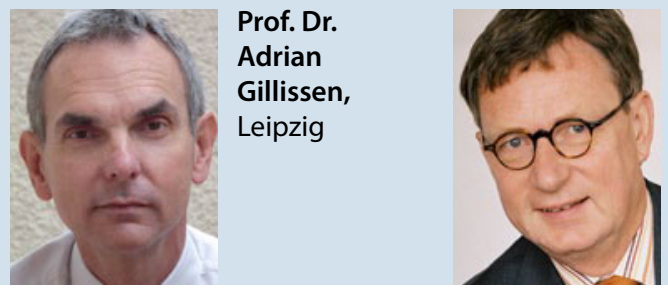

Prof. Dr. med. Thomas Katzorke, Essen

\section{Protonenpumpeninhibitoren: bei Säuglingen mit Reflux nicht wirksam?}

Die gastroösophageale Refluxkrankheit (GÖRK) bei Kindern macht nicht nur den betroffenen Familien, sondern auch den Ärzten erhebliche Probleme sowohl diagnostisch als auch therapeutisch. Nach dem Rückzug des Prokinetikums Cisaprid vor einigen Jahren wird standardmäßig mit Protonenpumpeninhibitoren (PPI) behandelt.

$\mathrm{D}$ ie vorliegende systematische Übersicht von van der Pol et al. aus Amsterdam untersuchte die Evidenz für eine PPI-Therapie bei Säuglingen und Kindern im Alter zwischen 0 und 17 Jahren. Die Arbeitsgruppe identifizierte zehn randomisierte prospektive Studien, und zwei Studien mit Crossover-Design hatten die erforderliche methodische Qualität. Folgende PPI wurden angewendet: Omeprazol (4), Esomeprazol (2), Lansoprazol (3) und Pantoprazol (3). Als Kontrollen dienten hydrolysierte Formulanahrung, Placebo, Alginat oder Ranitidin.

In den fünf Studien bei Neugeborenen und Säuglingen fand sich gegenüber Placebo weder eine signifikante Verbesserung der Refluxsymptome (Schreien, Spucken) noch der pH-Metrie; histologische Verlaufskontrollen wurden nicht dokumentiert.

In den fünf Studien bei Klein- und Schulkindern kamen keine Placebokontrollen, sondern andere Antirefluxmedikamente oder unterschiedliche Dosierungen der PPI zum Einsatz. In allen Fällen wurden die Symptome durch PPI gleich effektiv beeinflusst, mit einer signifikanten Verbesserung gegenüber den Ausgangsbefunden nach allen Maßnahmen. Die Säureproduktion des Magens, nicht aber histologische oder endosko- pische Scores wurde durch PPI effektiver reduziert als mit Aliginat oder Ranitidin.

Die zwei Studien bei Adoleszenten verglichen unterschiedliche Dosierungen von PPI; in allen Gruppen verbesserten sich die Refluxsymptome unabhängig von der Dosis.

Nebenwirkungen waren meist mild und vorübergehend; bemerkenswert ist vor allem die signifikante Erhöhung von Atemwegsinfekten in der PPI-Gruppe bei Säuglingen.

Die Autoren kommen zu dem Schluss, dass aufgrund des fehlenden Beweises der Effektivität und des fehlenden langfristigen Unbedenklichkeitsnachweises vor allem bei Säuglingen PPI nicht eingesetzt werden sollten.

Van der Pol RJ et al. Efficacy of proton-pump inhibitors in children with gastroesophageal reflux disease: A systematic review. Pediatrics 2011; 27: 925-35

Kommentar: Normalerweise werden an dieser Stelle Originalarbeiten besprochen. Wegen der Wichtigkeit des Themas haben wir für das Review von van der Pol et al. eine Ausnahme gemacht. Auch wenn der Einsatz der PPI mittlerweile als Standard auch für Kinder ab Geburt wahrgenommen wird, sollte dieses Vorgehen neu hinterfragt werden. Vor

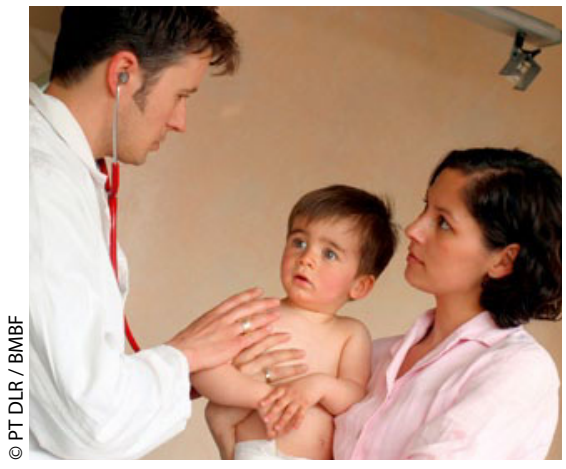

Bei Kleinkindern mit gastroösophagealer Refluxkrankheit herrscht oft Ratlosigkeit.

allem gibt es für die weit verbreitete probatorische Therapie, die zum Beweis einer Refluxkrankheit dienen soll, keinerlei Evidenz. An die hohe Rate spontaner Besserungen des Refluxproblems bei Säuglingen und Kleinkindern durch Maturation des Sphinkters sei erinnert. Auch eine lang dauernde Therapie mit PPI birgt bezüglich der potenziellen $\mathrm{Ne}$ benwirkungen möglicherweise unbekannte Risiken. Die Schlussfolgerungen führen zu einer gewissen Ratlosigkeit, wie nun bei gesicherter Diagnose konkret behandelt werden sollte, besonders wenn riskante Folgen wie Apnoe, Asthma oder Gedeihstörung beziehungsweise eine schwere Ösophagitis bestehen. Insofern sollten die Folgen des Refluxes sauber dokumentiert werden und wenn man sich doch zu einer Therapie mit PPI entscheidet, der Erfolg auch kontrolliert werden. Dringend notwendig sind eine intensivere Erforschung der Ursachen (nicht der Verschlussdruck des unteren Ösophagussphinkters scheint die entscheidende Determinante des Refluxgeschehens, sondern transiente Relaxationen des Sphinkters) und gute prospektive, placebokontrollierte Studien zur Therapie. Dr. Martin Claßen 